\title{
How to Build A Strong Theoretical Foundation*
}

\author{
Paul E. Smaldino \\ Department of Cognitive \& Information Sciences \\ University of California, Merced \\ psmaldino@ucmerced.edu
}

It is our theories that shape how we see the world and the questions we ask of it. Fried argues that psychological science is plagued by weak theories, and that there is a real need for building and testing strong theories that include formal models. I agree. Fried calls for better training in the construction of formal theory, and I enthusiastically agree with this as well (see Smaldino, 2020a; 2020b). However, I am concerned that the road to establish such a training in program will be long and hard. Fried ends his piece with tentative optimism, but little in the way of concrete proposals.

In my experience, training in psychological science tends to involve learning a broad historical overview of the field, conceptual exposure to key ideas and findings, and deep training in the methodologies of one's subfield. This is probably a good system for the stabilization of particular norms, but it is less than ideal for building up a community with a strong theoretical foundation. A few years ago, I gave a workshop on formal modeling at a European university. Afterwards, one of the faculty said to me something like "I can clearly see the value in this sort of modeling. In psychology, we receive zero training in how to even think like this.” Changing this fact will require more than polemics on the value of modeling or the offering of a few workshops. It will require substantial changes to the disciplinary norms of psychological science.

${ }^{*}$ Commentary on E. Fried, "Lack of theory building and testing impedes progress in the factor and network literature." https://psyarxiv.com/zg84s/ 
Here I'll outline what I think some of the necessary changes are and why implementing them will be challenging. I'll conclude with some thoughts on how to overcome those challenges. Constructing good strong theories requires integration of the skills currently possessed by psychological scientists with (1) increased interdisciplinarity, (2) increased technical prowess, and (3) increased philosophical scrutiny.

\section{Increasing Interdisciplinarity}

I was once at a conference where a social psychologist gave a presentation about how people often equate the social norms they are raised with the way things should be. Let's ignore any potential concerns about the replicability, generalizability, or cultural myopia of this claim and assume that it is true. It might well be. The bigger problem is that the speaker lamented about simply terrible this was, and how their method of intervention could help people to reliably question social norms and explore alternative ways of being. I am doubtful that their method, which was along the lines of having people reading an essay about creative thinking, had any real long term effects, but again, this is not my primary concern. I am more concerned with the naïve view of human social behavior. The psychologist was stuck in an individualistic, Western, upper-middle-class mindset and apparently assumed that independent thought, iconoclastic behavior, and norm violations were universally desirable traits. Not once was there any consideration of the instrumental function of social norms (North, 1990; Boyd \& Richerson, 2002; Hawkins et al., 2018), the societal or evolutionary benefits that a psychology predisposed toward following norms might confer (Simon, 1990; O’Gorman et al., 2008; Chudek \& Henrich, 2011; Gelfand et al., 2020), or the logical consequences of having a society comprised entirely of free thinkers. I thought of the anthropologists and other social scientists I know, and what they 
would make of this perspective that lacked an appreciation of culture and social relationships. I thought of the biologists and complexity scientists I know, and what they would make of this perspective that lacked an appreciation of emergent phenomena and collective behaviors. I thought of how if this psychologist had even once presented their work in front of nonpsychologists, these concerns could have been voiced and dealt with.

I don't mean to pick on one social psychologist. The issue is far broader. If we are interested in behavior or cognition, we have to acknowledge that individuals don't simply exist as isolated entities, but are part of a larger system that has historical, developmental, and social entanglements. Different parts of the story are the focus of different research communities, and this division of labor is necessary to investigate any question deeply, but its value is maximized only when there is at least occasional interchange between those communities.

You cannot form a strong theory of human psychological processes without at least some understanding of humans as social, cultural, and biological creatures. And you cannot understand complex processes without at least some understanding of how complex dynamics unfold. This does not mean that everyone must become an expert in everything; obviously that isn't reasonable. What is does mean is that we need learn from other disciplines and to proactively allow ourselves to be evaluated by interested parties with different disciplinary backgrounds. This exchange will strengthen our theories by subjecting them to scrutiny from all sides. Other fields have also done a lot of the work on certain questions, and there is no reason to reinvent the wheel.

Developing comfort with interdisciplinary can be challenging. It involves reading additional literatures, each of which may have different jargon, theoretical foundations, and methods. It involves cultivating additional social relationships. And it might mean confronting 
pathologies in your home discipline that are normative there but seen as problematic in other communities. All of this can be time consuming and otherwise effortful. These hurdles must be overcome, however, if we are ever to have an integrated science of behavior-and I hope that we do (cf. Nettle, 2018; Smaldino, 2019).

\section{Increased Technical Prowess}

Formalizing theories with models often requires comfort with advanced computational and mathematical techniques, as well as familiarity with a wide range of phenomena and concepts in mathematics, dynamical systems, and complexity science. Many psychologists have never had a course in calculus, let alone dynamical systems, and it is likely that only a small minority could explain the differences between small-world and scale-free networks ${ }^{2}$. If strong theories supported by formal models are going to become an integral part of psychological science, the field is going to have to become more technically sophisticated.

Psychologists are hardly methodological simpletons. More and more early- and midcareer researchers have legitimate programming skills and are familiar with advanced statistical techniques such as Bayesian inference and causal inference. Indeed, statistical training for psychologists has been continually improving over the last decade. This is a good thing. Yet statistics are not sufficient. As Fried rightly points out, statistical models are not theoretical models of how processes are generated. Developing the latter sort of model takes different skills than does the former. And psychological scientists are still not getting adequate training on how this is done.

\footnotetext{
${ }^{2}$ Incidentally, it is noteworthy that Fried focuses on network models and factor models as two distinct techniques, when exploratory factor analysis is essentially a type of network community detection algorithm.
} 
Now we are faced with two problems. First, there is the issue of time. A person can only invest so much time learning concepts and techniques. It is impractical to really learn it all. The second is the issue of temperament. Many good experimentalists are middling mathematicians. The reverse is also true. If we expect psychologists to be skilled in the lab, at analyzing data, and at building formal models, we will likely lose many valuable contributions from those who are good at only one or two of these. This is where Fried's point about theoretical psychology is so important. I was once told by a psychology professor that if a person was not conducting laboratory experiments, they were not a psychologist. This attitude, to the extent it is widespread, has to change. Departments of psychology should be eager to employ theorists, just as departments of economics, biology, and physics have been ${ }^{3}$. To some extent, theorists and modelers have had some success in adjacent fields like neuroscience (Dayan \& Abbott, 2001) and cognitive science (Farrell \& Lewandowsky, 2011), and the newer interdisciplinary fields of cultural evolution, human behavioral ecology, and computational social science have all been strongly influenced by model-driven theory (Boyd \& Richerson 1985; Kennett \& Winterhalder 2006; Macy \& Willer, 2002). Although there are examples of researchers using formal models in psychology that date back several decades (e.g., Rescorla \& Wagner, 1972; Kalick \& Hamilton, 1986; Rumelhardt et al. 1986; Nowak et al., 1990), mainstream psychology still lags behind other disciplines in its embrace of strong theories supported by formal models.

If specialization in theory development and model building are supported, psychologists interested in stronger theories will not face the impossible task of having to become experts in an overwhelmingly wide array of technical traditions. Greater support for theoretical psychology also allows psychology to be defined by the topic of study, not the technique. Formal models of

\footnotetext{
${ }^{3}$ Your mileage may vary. Given the current state of the academic job market, it is not easy to get a job as a theorist in any field.
} 
psychological phenomena have been developed and will continue to be developed, but this work is often done in departments of physics, mathematics, and computer science. Perhaps the field of psychology has been so thoroughly devastated by the problems of replicability and generalizability that its lessons should discarded, and a replacement field should be built anew on firmer foundations. In that case, perhaps this new field should arise from fields like computer science and physics. On the other hand, if psychological scientists remain confident that their field has something concrete to contribute, that the expertise of experimental psychologists has value to inform strong theories of mind, brain, and behavior, then theory development should happen among the psychologists, to be informed by and assessed by those closest to the subject. In other words, psychology departments need to hire modelers and support training in modeling, and allow theoretical psychology to blossom as a legitimate subfield.

\section{Increased Philosophical Scrutiny}

Learning to develop formal models involves more than technical prowess. It also requires an artful approach to parsing the world to create meaning and scientific value. A common mistake when considering a model of some phenomenon is to assume that, despite the warnings of George $\mathrm{Box}^{4}$, there exists, somewhere, a capital-T True model of the data-generating process, and that the only roadblock to discovering that True model is the presence of noise in the data collection process (Fodor, 1987; Devezer et al., 2019). This is silly. The patterns we see in the world are bits of information, encoded and chunked by machines of the model type Homo sapiens. The data are the way they are because of the way we parse the world (Dennett, 1991). This is perhaps why the difficulties in establishing training in formal theory will be greater than

\footnotetext{
4 "All models are wrong; some are useful."
} 
in establishing other methodological courses. Developing theory with formal models is not the execution of an algorithm, but a reckoning with one's conceptualization of reality.

Fried writes, "To escape vagaries of language and imprecise predictions, network theory should define what a symptom is, and provide a complete list of all symptoms, causal symptom relations, time-frames, underlying mechanisms, and conditions (e.g. moderators) under which these occur." This sounds great. Models help us to be more precise and skirt the inherent ambiguity of language (Smaldino, 2017; 2020b). But we also can go down a rabbit hole of taxonomy in an effort to be maximally precise and not leave anything out. All symptoms? Do we need to include a slight twitch of the eyebrow, or gastrointestinal distress? All causal relations? Do we need to include the effects of watching a scary movie, or eating cheese when lactose intolerant? All time-frames? From the nanoseconds to decades? We always leave some things out. A map containing all the detail of the territory is useless. The question is whether we are leaving out the appropriate things to best answer our questions, and whether our questions are the best ones we can be asking.

Philosophy has been defined many ways, but in my experience it is a discipline fixated on taking seriously questions of the type "What exactly are we talking about when we talk about $X$ ?" Being open to this sort of philosophical question is imperative for building strong theories. A model of some system must be designed in service of explaining or proposing some phenomena. This is a critical point, because our explananda will determine the questions we ask, and therefore the nature of the models we build.

To develop a theory of some phenomenon, we must first decompose our system into a set of relevant parts, and then specify the potential properties of those parts, the relationships between the parts, and the temporal dynamics by which those properties and relationships can 
change $^{5}$ (Smaldino, 2020b). Selecting the appropriate parts, properties, and relationships is nontrivial. It is also not a matter of selecting those that correspond to the True theory. There is no True theory, only theory that helps understanding and/or prediction with better or poorer precision and generalizability. Within a given disciplinary tradition, describing a phenomenon often involves an implicit and presumably shared decomposition of a particular system into parts, properties, and relationships. This decomposition is the set of assumptions regarding what the things being studied are, at least insofar as we need to know to answer our research questions. New theories can foment paradigm shifts by giving us more useful decompositions.

For almost any system of interest to psychological scientists, the parts are emergent properties, not natural kinds. Fried notes this in his discussion of network models of psychological constructs. But the point is broader: it is necessarily true of all psychological constructs. Parts must be meaningful insofar as they can be identified, classified, and ideally, measured. Sometimes this is easy — for vertebrates, at least, it is usually easy to say what is or is not an individual organism. Other times, this can be difficult. What is a behavior? What is a gene? What is a psychological construct like intelligence, fear, or the concept of "squishy"? To build formal models, we must operationalize these in terms of their effect on the parts of the system and the relationships between those parts. We must be willing to scrutinize our characterizations of what we are talking about.

A related point is the reconsideration of what a scientific finding is. A question I often get asked as a modeler is: "What data do you work with?" Sometimes, there is a concrete answer, when I am building models to explain specific patterns in specific data sets. Other times, I am exploring the consequences of particular decompositions. What happens when I conceptualize

\footnotetext{
${ }^{5}$ I have developed this idea over a number of years, but it is greatly influenced by Simon (1962), Kauffman (1971), and Wimsatt (1974).
} 
the world as a particular set of parts, properties, and relationships? This can be important foundational work in the development of strong theory, and it is an error to suppose that all models should be in the service of explaining specific empirical data. A key point we must ask concerns the extent to which the assumptions of a model reflect reasonable assumptions about the forces shaping our phenomenon? If they do not, and the model is sufficiently complex and/or underspecified, then we may fit end up with good fits of a poor model that tell us little about underlying generative processes.

Fried illustrates this point well (see also Robinaugh et al., 2020). For another striking example, consider the partner-choice models of Kalick and Hamilton (1986) and Hills and Todd (2008). Both papers concerned the processes used by individuals to make decisions in seeking out and selecting long-term romantic partners. They compared formalizations of different partner-choice decision rules and purported to show support for particular decision rules, based on their models' fit to empirical data on actual romantic partnerships. However, both models made several questionable assumptions, including (1) that individuals encounter potential partners at random, and (2) that preference strengths varied with the cube of their distance from the ideal. Jeff Schank and I showed that by altering these assumptions_-by imposing network structures and altering the preference functions without changing their monotonicity —almost any decision rule considered could be made to fit the empirical data (Smaldino \& Schank, 2012). Here, the point of our modeling was not to fit a theory to data, but to explore the implications of various assumptions, and to illustrate the perils in rushing to fit an underspecified model to data. As a solution to the problem of underspecification, Grimm et al. (2005) have suggested a "pattern-oriented" approach, whereby models should be judged in part by their fit to multiple aspects of the study system. 
Psychologists need to scrutinize how they decompose their systems to answer their research questions - as well as considering how their questions are shaped by those decompositions. Encouragingly, psychologists are increasingly engaging with philosophy of science in the aftermath of the replicability crisis, but they should also be encouraged to engage more directly with philosophy of mind, philosophy of biology, and philosophy of modeling. Philosophical scrutiny is further impeded by the fact that publishing and grants are still the coin of the realm in academic science (Smaldino \& McElreath, 2016). Many top journals are reluctant to publish purely theoretical work, and funding for such research is often hard to come by (purely theoretical work can also be done at little cost, which should be seen as a benefit given budget constraints, but also means that theorists may bring in fewer or smaller grants). Theoretical work is time-consuming, and will rarely generate splashy, newsworthy results. Either journals must become more open to formal theory or new theoretically-oriented journals must arise. Or perhaps the reification of publication and grants as measures of quality will subside, and more holistic evaluations will dominate hiring and promotion committees. We can hope, anyway.

\section{Making It Happen}

Norm change is hard when norms are entrenched in a community. I worry that the sort of hand-waving explanations characteristic of weak theories are so deeply entrenched in norms of psychological science that the current gatekeepers will simply not allow a transition to happen without a fight. In that case, we will have to fight for it.

What can be done? Recruit people to the cause. Demonstrate the tangible benefits of stronger theories. Demand better of our peers. Judge work not by the normative standards of a 
subfield, but by the best work that is being on the subject coming from any field. Be more interdisciplinary. Remember that almost every topic tackled by researchers working in psychology departments is also worked on by researchers working in departments of anthropology, sociology, political science, economics, communication, biology, neuroscience, cognitive science, or computer science. Support greater technical prowess with the training and hiring of theoreticians. Advances in statistical training represents a good direction. But if we are serious about incorporating formal modeling into psychological science, we need to encourage a technical subfield in which at least some psychologists concentrate primarily on theory. Scrutinize how a theory decomposes the world into parts, properties, and relationships. Question the assumptions made by those theories to tear them down and build better ones. Let's build a strong theoretical foundation for the study of human behavior.

\section{References}

Boyd, R., \& Richerson, P. J. (1985). Culture and the evolutionary process. University of Chicago Press.

Boyd, R., \& Richerson, P. J. (2002). Group beneficial norms can spread rapidly in a structured population. Journal of Theoretical Biology, 215(3), 287-296.

Chudek, M., \& Henrich, J. (2011). Culture-gene coevolution, norm-psychology and the emergence of human prosociality. Trends in Cognitive Sciences, 15(5), 218-226.

Dayan, P., \& Abbott, L. F. (2001). Theoretical neuroscience: Computational and mathematical modeling of neural systems. MIT Press.

Dennett, D. C. (1991). Real patterns. Journal of Philosophy, 88, 27-51. 
Devezer, B., Nardin, L. G., Baumgaertner, B., \& Buzbas, E. O. (2019). Scientific discovery in a model-centric framework: Reproducibility, innovation, and epistemic diversity. PLOS ONE, 14(5), e0216125.

Farrell, S., \& Lewandowsky, S. (2011). Computational modeling in cognition: Principles and practice. Sage.

Fodor, J. A. (1987). Psychosemantics: The problem of meaning in the philosophy of mind. MIT Press.

Gelfand, M. J., Jackson, J. C., Pan, X., Nau, D., Dagher, M. M., Lange, P. V., \& Chiu, C. (2020). The importance of cultural tightness and government efficiency for understanding COVID-19 growth and death rates. PsyArXiv. https://psyarxiv.com/m7f8a.

Grimm, V., Revilla, E., Berger, U., Jeltsch, F., Mooij, W. M., Railsback, S. F., et al. (2005) Pattern-oriented modeling of agent-based complex systems: Lessons from ecology. Science, 310, 987-991.

Hawkins, R. X., Goodman, N. D., \& Goldstone, R. L. (2019). The emergence of social norms and conventions. Trends in Cognitive Sciences, 23(2), 158-169.

Hills, T., \& Todd, P. (2008). Population heterogeneity and individual differences in an assortative agent-based marriage and divorce model (MADAM) using search with relaxing expectations. Journal of Artificial Societies and Social Simulation, 11(4), 5.

Kalick, S. M., \& Hamilton, T. E. (1986). The matching hypothesis reexamined. Journal of Personality and Social Psychology, 51(4), 673-682.

Kauffman, S. A. (1971). Articulation of parts explanation in biology and the rational search for them. In R. C. Buck \& R. S. Cohen (Eds.), PSA 1970 (pp. 257-72). Irvine, CA: Philosophy of Science Association. 
Kennett, D. J., \& Winterhalder. (2006). Behavioral ecology and the transition to agriculture. University of California Press.

Macy, M. W., \& Willer, R. (2002). From factors to actors: Computational sociology and agentbased modeling. Annual Review of Sociology, 28(1), 143-166.

Nettle, D. (2018). What we talk about when we talk about biology. In Hanging on to the edges: Essays on Science, Society and the academic life. Open Book Publishers.

North, D. C. (1990). Institutions, institutional change and economic performance. Cambridge University Press.

Nowak, A., Szamrej, J., \& Latané, B. (1990). From private attitude to public opinion: A dynamic theory of social impact. Psychological Review, 97(3), 362.

O'Gorman, R., Wilson, D. S., \& Miller, R. R. (2008). An evolved cognitive bias for social norms. Evolution and Human Behavior, 29(2), 71-78.

Rescorla, R. A., \& Wagner, A. R. (1972). A theory of Pavlovian conditioning: Variations on the effectiveness of reinforcement and non-reinforcement. In A. H. Black \& W. F. Prokasy (eds.), Classical conditioning II: Current research and theory. Appleton-Century-Crofts.

Robinaugh, D., Haslbeck, J., Ryan, O., Fried, E. I., \& Waldorp, L. (2020). Invisible hands and fine calipers: A call to use formal theory as a toolkit for theory construction. PsyArXiv. https://psyarxiv.com/ugz7y

McClelland, J. L., Rumelhart, D. E., \& PDP Research Group. (1986). Parallel distributed processing. Explorations in the microstructure of cognition. MIT Press.

Simon, H. A. (1962). The architecture of complexity. Proceedings of the American Philosophical Society, 106, 467-482. 
Simon, H. A. (1990). A mechanism for social selection and successful altruism. Science, 250, $1665-1668$.

Smaldino, P. E. (2017). Models are stupid, and we need more of them. In R. Vallacher, S. J. Read, \& A. Nowak (Eds.), Computational social psychology (pp. 311-331). New York: Psychology Press.

Smaldino PE (2019) A modeling approach that integrates individual behavior, social networks, and cross-cultural variation. Trends in Cognitive Sciences, 23, 818-820.

Smaldino, P. E. (2020a). Evolutionary social science needs programmatic training in how models work. Evolution and Human Behavior, in press.

Smaldino, P. E. (2020b). How to translate a verbal theory into a formal model. Social Psychology, in press.

Smaldino, P. E., \& McElreath, R. (2016). The natural selection of bad science. Royal Society Open Science, 3, 160384.

Smaldino, P. E., \& Schank, J. C. (2012). Human mate choice is a complex system. Complexity, 17(5), 11-22.

Wimsatt, W. C. (1974). Complexity and organization. In K. Schaffner \& R. S. Cohen (Eds.), PSA 1972 (pp. 67-86). Irvine, CA: Philosophy of Science Association. 